\title{
Refining bioreactor design using autoclavable glass bonding*
}

\author{
Cornelia Bähr ${ }^{1}$, Elisabeth Stammen ${ }^{2}$, Sarah Zahlten ${ }^{1}$, Stefan Böhm ${ }^{2,3}$, Klaus Dilger ${ }^{2}$, Jochen Büchs ${ }^{1}$ \\ ${ }^{1}$ AVT-Biochemical Engineering, RWTH Aachen University, Aachen, Germany; \\ ${ }^{2}$ Institute of Joining and Welding, Technische Universität Braunschweig, Braunschweig, Germany; \\ ${ }^{3}$ Institute of Production Technology and Logistics, Kassel University, Kassel, Germany. \\ Email: jochen.buechs@avt.rwth-aachen.de
}

Received 31 May 2011; revised 1 July 2011; accepted 13 July 2011.

\begin{abstract}
Selecting the right design of bioreactors is crucial for guaranteeing the reproducibility of bioprocesses. Up to now, conventionally designed bioreactors consist typically of melted or clamped joints. Since melting of borosilicate glass leads to large deformed areas along the joint, the desired geometric reproducibility is not ensured. Moreover, clamping complicates and greatly restricts the bioreactor design. Bonding, however, is advantageous in that it does not alter the material joined and it is easy to use. Furthermore, it has been recently shown that specially developed glass bonding techniques withstand multiple autoclaving cycles. The current research investigated practicerelevant parameters influencing the lifespan of epoxy- or (urethane) acrylate-bonded glass bioreactors. Hereby, the influence of cleaning and sensitivity to fermentation compounds (ethanol and acetic acid) was quantified using glass-glass and glass-stainless steel specimens. Whereas cleaning did not adversely affect the durability of glass bonds, high concentrations of the fermentation compounds ethanol and acetic acid resulted in accelerated corrosion and subsequent bond failure. Moreover, no effect of eight different epoxy and (urethane) acrylate adhesives was observed on selected model organisms Escherichia coli K12 and Hansenula polymorpha wild type. Another objective of this study was to refine the design of two small-scale bioreactors (ca. $250 \mathrm{~mL}$ ) by replacing clamps and melted joints by adhesive joints. It was found that the bonded bioreactors yielded a higher geometric reproducibility than that of conventional melted or clamped ones. In conclusion, bonded glass joints greatly enhance the geometric reproducibility of bioreactors and, in turn, the reproducibility of bioprocesses. As glass bonding is easy to handle, it opens up new opportunities to design bioreactors that had been previously too expensive and complicated.
\end{abstract}

*German Federation of Industrial Research Associations "Otto von Guericke” e.V. (AiF)
Keywords: Bioreactor; Durability; Borosilicate Glass; Stainless Steel 1.4301; Epoxy Adhesive; Acrylate Adhesive

\section{INTRODUCTION}

Bioreactor design essentially defines power input, mass transfer, mixing, and, thus, process performance. In particular, for shaken bioreactors, the shape of the reactor wall affects the shape of the rotating liquid and, hence, the mass transfer area [1-4]. Shaken bioreactors are typically applied in screening for optimal strain or process conditions [1,5-8], whereby the differences in performance between the strains and/or conditions are often marginal. Therefore, it is crucial to ensure precise and reproducible environmental conditions for the cultured microorganisms which, in turn, guarantee the reproducibility of bioprocesses. Hereby, the geometry of the bioreactors plays a key role [1,9-11].

Currently, most bioreactors for screening are made of glass, allowing easy visual checking and optical monitoring [1,12-14]. However, the conventional glass joining technologies are problematic. The melting of glassglass joints lacks geometric reproducibility, because the glass substrate becomes soft in large areas along the joint, resulting in large deformations. Moreover, clamping glass to other materials such as stainless steel, most frequently applied in bioreactors, is complicated, and restricts the whole design. In addition, clamping can lead to gaps at the joint, increasing the risk of contamination of the bioprocess. Since glass is brittle, the clamping exacerbates stress fractures, resulting in fatigue failure at an early stage of use. Furthermore, the different thermal expansions of glass and stainless steel cause problems. All these factors basically reduce the reproducibility and efficiency of experiments in glass bioreactors.

Unlike the conventional glass joining technologies, adhesive bonding presents numerous advantages. Bonding homogeneously distributes stresses. Moreover, the adhesive is easy to apply, has low weight and is inex- 
pensive [15-17]. It has been proven that glass adhesive bonds can withstand multiple autoclaving cycles [18].

Nevertheless, the lifespan of bonded glass in bioreactors is not only defined by the long-term durability of the materials but also by practice-relevant parameters such as cleaning, fermentation compounds, and the actual cultivated microorganisms. Thus, this current paper addresses this question. Moreover, in order to improve the reproducibility of experiments in small-scale bioreactors, another principal aim of the current study is to refine the design of two small-scale bioreactors (ca. $250 \mathrm{~mL}$ ) in terms of enhancing the geometric reproducibility and simplifying their design.

\section{MATERIALS AND METHODS}

\subsection{Materials}

Materials tested were borosilicate glass Duran 3.3 ISO 3585 (Duran Group GmbH, Mainz/Germany) and stainless steel 1.4301 (LKT GmbH, Aachen/Germany). As adhesives, the two-component epoxy M-31 CL (HenkelLoctite, Garching/Germany) and the UV-curable urethane acrylates GB 368 (Delo Industrial Adhesives, Windach/Germany) and Vitralit 7563 (Panacol-Elosol, Oberursel/Germany) were applied.

\subsection{Curing Procedure}

All surfaces were degreased with 2-butanone ("MEK" for the non-scientific name methyl-ethyl-ketone) before application of the adhesive. To possibly improve the bond durability, part of the glass and steel components was additionally pre-treated with Pyrosil ${ }^{\circledR}$ [18]. The curing cycles applied to the adhesives were recommended by the manufacturers (confer to Table 1). The M-31 CL was cured at ambient temperature for 5 days or thermally at $65^{\circ} \mathrm{C}$ for $2 \mathrm{~h}$. In all cases, the adhesive thickness was controlled at $0.2 \mathrm{~mm}$ by glass beads (Sigmund Lindner GmbH, Warmensteinach/Germany).

\subsection{Accelerated Aging by Autoclaving}

For accelerated aging tests, shear specimens were produced according to a previous study [18]. The aging of the specimens was accelerated in at least 25 standard cycles of autoclaving (20 minutes at $121^{\circ} \mathrm{C}, 2 \mathrm{bar}$, saturated steam) in a D-65 autoclave (Systec, Wettenberg/ Germany). The shear strength was measured at ambient temperature (RT) according to Bähr et al. [18].

\subsection{Investigation of Practice-Relevant Parameters}

\subsubsection{Cleaning}

Specimens made of borosilicate glass/borosilicate glass ("bor-bor") and borosilicate glass/stainless steel ("bor-st”) were bonded with the urethane acrylate GB 368 or the
Table 1. Adhesives tested concerning the influence on model microbes.

\begin{tabular}{|c|c|c|c|}
\hline Adhesive & $\begin{array}{l}\text { Manufac- } \\
\text { turer }\end{array}$ & $\begin{array}{l}\text { Chemical } \\
\text { structure }\end{array}$ & Curing \\
\hline GB 310 & $\begin{array}{c}\text { Delo } \\
\text { (Windach, D) }\end{array}$ & Acrylate & $\begin{array}{c}\text { UV } 320-400 \\
n m, 20 s\end{array}$ \\
\hline GB 368 & $\begin{array}{c}\text { Delo } \\
\text { (Windach, D) }\end{array}$ & $\begin{array}{l}\text { Urethane } \\
\text { acrylate }\end{array}$ & $\begin{array}{c}\text { UV } 320-420 \\
n m, 15 \text { s }\end{array}$ \\
\hline $1-20537$ & $\begin{array}{c}\text { Dymax } \\
\text { (Frankfurt, D) }\end{array}$ & $\begin{array}{c}\text { Urethane } \\
\text { (meth) acrylate }\end{array}$ & $\begin{array}{c}\text { UV } 300-500 \\
n m, 20 s\end{array}$ \\
\hline $\begin{array}{l}\text { Vitralit } \\
6128\end{array}$ & $\begin{array}{c}\text { Panacol-Elosol } \\
\text { (Oberursel, D) }\end{array}$ & Acrylate & $\begin{array}{c}\text { UV-A } 60 \\
\mathrm{~mW} / \mathrm{cm}^{2}, 15 \mathrm{~s}\end{array}$ \\
\hline $\begin{array}{l}\text { Vitralit } \\
7563\end{array}$ & $\begin{array}{l}\text { Panacol-Elosol } \\
\text { (Oberursel, D) }\end{array}$ & Acrylate & $\begin{array}{c}\text { UV-A } 40 \\
\mathrm{~mW} / \mathrm{cm}^{2}, 15 \mathrm{~s}\end{array}$ \\
\hline M-31CL & $\begin{array}{l}\text { Henkel-Loctite } \\
\text { (Garching, D) }\end{array}$ & $\begin{array}{l}\text { Two-component } \\
\text { epoxy }\end{array}$ & $5 \mathrm{~d}, \mathrm{RT}$ \\
\hline $\begin{array}{l}\text { Epo-Tek } \\
353 \text { ND }\end{array}$ & $\begin{array}{c}\text { Polytec PT } \\
\text { (Waldbronn, D) }\end{array}$ & $\begin{array}{l}\text { Two-component } \\
\text { epoxy }\end{array}$ & $\begin{array}{c}48 \mathrm{~h}, \mathrm{RT}+1 \mathrm{~h}, \\
120^{\circ} \mathrm{C}+1 \mathrm{~h} \\
150^{\circ} \mathrm{C}\end{array}$ \\
\hline $\begin{array}{l}\text { EPVP } \\
4611 / 4\end{array}$ & $\begin{array}{c}\text { Polytec PT } \\
\text { (Waldbronn, D) }\end{array}$ & $\begin{array}{c}\text { Two-component } \\
\text { epoxy }\end{array}$ & $16 \mathrm{~h}, \mathrm{RT}$ \\
\hline
\end{tabular}

two-component epoxy M-31 CL after degreasing with MEK or an additional Pyrosil ${ }^{\circledR}$ pre-treatment. The M-31 CL was cured as recommended by the manufacturer as well as cured thermally (Section 2.2).

Since bioreactors with glass bonds are typically applied in biotechnological and medical applications, the following two standard cleaning programs were chosen (Miele \& Cie. KG, Gütersloh/Germany): SM1 to clean laboratory equipment and SM2 to clean medical devices such as surgical instruments. These standard programs were adapted to meet worst-case scenarios in terms of temperature and the concentration of cleaning agents. The modifications applied were: 1) utilization of $1 \%$ cleaner containing active chlorine (HOCl) in SM1, 2) increased concentration $(0.5 \%)$ of phosphoric acid for neutralization (normally $0.1 \%-0.3 \%$ ) in both cleaning programs. 3) SM1 is characterized by two alkaline cleaning phases while common cleaning procedures for such laboratory equipments contain only one. In SM1, the cleaning material was exposed to maximal temperatures of $80^{\circ} \mathrm{C}$ during cleaning phase and $85^{\circ} \mathrm{C}$ during final rinse. In SM2, the cleaning material was exposed to more ambient temperatures $<60^{\circ} \mathrm{C}$ during cleaning phases even to $93^{\circ} \mathrm{C}$ during the subsequent thermal disinfection.

Ten specimens of each substrate/adhesive/pre-treatment combination were cleaned in 50 cycles of SM1or SM2. After cleaning, five specimens were additionally aged in 50 standard autoclaving cycles (Section 2.3). The shear strength was measured (Section 2.3) immediately after cleaning or after the additional autoclaving.

\subsubsection{Loading during Autoclaving}

The bond durability under load during autoclaving was investigated, because the equipment is typically loaded with other devices during actual autoclaving e.g. in a tabletop autoclave. To simulate a realistic scenario, spe- 
cimens made of bor-bor and bor-st (bonded with GB 368 and pre-treated by MEK degreasing or additionally treated with Pyrosil ${ }^{\circledR}$ ) were autoclaved under load of $143 \mathrm{~g}$, which is the weight of a RAMOS flask equipped with caps (confer Section 2.5.1). The load of such specimens was realized as depicted in Figure 1.

\subsubsection{Fermentation Compounds}

Since there are many fermentation compounds in bioprocesses, the investigated substances were restricted to ethanol and acetic acid. Both ethanol and acetic acid are highly reactive and common in microbial processes (ethanol as target product reviewed in [19-22]; acetic acid as target product in [23-26]). Therefore, bor-bor and bor-st specimens bonded with GB 368 or M-31 CL were autoclaved both in 25 and 50 standard cycles, whereby they were immersed in a $120 \mathrm{~g} / \mathrm{L}(15 \%(\mathrm{w} / \mathrm{v}))$ ethanol/200 g/L acetic acid (20\% (w/v)) solution. The concentrations of ethanol and acetic acid were chosen as the maximal concentrations found in the literature for microbial processes [27-29]. To guarantee almost constant concentrations of ethanol and acetic acid during the autoclaving, the solutions were renewed every tenth cycle of steam sterilization. Afterwards, the shear strength of the bonded glass specimens was measured (Section 2.3).

The concentrations of ethanol and acetic acid were monitored using HPLC with an Organic Acid Resin HPLC pre-column $(40 \times 8 \mathrm{~mm})$ and Organic Acid Resin HPLC column $(250 \times 8 \mathrm{~mm})$ (both CS Chromatographie, Langerwehe/Germany). $5 \mathrm{mM}$ sulphuric acid was used as eluent (flow rate $0.6 \mathrm{~mL} / \mathrm{min}$ ). The ethanol or acetic acid was detected by UV diode array detector UVD 340S (Dionex Softron, Germering/Germany). Peak analysis and calculation was performed with Chromeleon software version 6.3 (Dionex Softron, Sunnyvale/USA).

\subsubsection{Model Microorganisms}

Since the applied adhesive could influence the cultivated

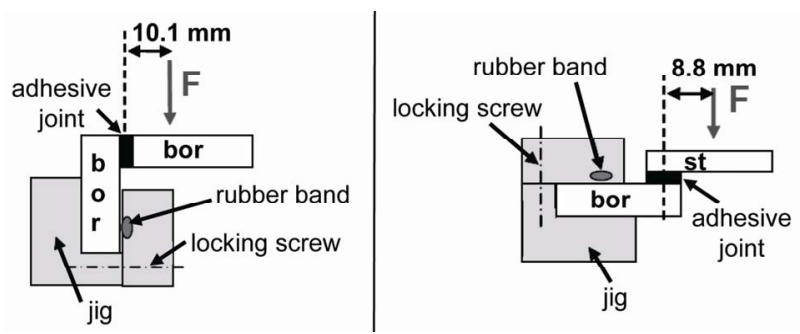

Figure 1. Investigation of bond durability under load during autoclaving. Specimens: borosilicate glass bonded to itself (bor-bor) or to stainless steel (bor-st) using the urethane acrylate adhesive Delo GB 368 degreased with 2-butanone or additionally pre-treated with Pyrosil@; autoclaving: 100 standard cycles (20 min at $121^{\circ} \mathrm{C}$ and 2 bar); specimens were loaded with $143 \mathrm{~g}$ (weight of a fully equipped RAMOS flask as one of the two bioreactors refined in this study). microbes, the bacterium Escherichia coli K12 (German Collection of Microorganisms 498) and the yeast Hansenula polymorpha wild type strain (German Collection of Microorganisms 70277) were cultivated in presence of disks made of pure adhesive. These cultivations were carried out in a Respiration Activity MOnitoring System (RAMOS) $[6,30]$ to monitor possible adverse effects of the adhesive on the metabolic activity of the microbes. RAMOS has already been successfully used for several investigations [6,30-35].

Adhesive disks (see Table 1) were produced as disks having a diameter of $15 \mathrm{~mm}$ and a height of $1 \mathrm{~mm}$. Immersed in deionized water, the specimens were sterilized in a standard autoclaving cycle. Then two disks were added to the microbial cultures. As positive controls, cultures without adhesive disks were conducted in parallel. After cultivation, the adhesive disks were washed with deionized water and optically checked with regard to changes of surface topology or color before the microbial cultures were sterilized to kill the microorganisms. Afterwards, the adhesive disks were washed and re-dried in a glass dish at ambient temperature for $24 \mathrm{~h}$. The color and the surface topology of the adhesive disks were documented 1) before and 2) after autoclaving to sterilize the disks, 3) after cultivation, 4) after that second autoclaving, and 5) after re-drying of the autoclaved disks.

Cultivation conditions: Both microorganism strains were cultivated in shake flasks in $10 \mathrm{~mL}$ mineral media at $37^{\circ} \mathrm{C}$, a shaking frequency of $350 \mathrm{rpm}$ and a shaking diameter of $50 \mathrm{~mm}$. E. coli $\mathrm{K} 12$ was cultivated in Wilms MOPS medium at $\mathrm{pH} 7.5$ [36,37] and $H$. polymorpha wild type in Syn6 MES medium at pH 6.4 [38] prepared according to Jeude et al. [39]. These cultures were inoculated with $4 \%(\mathrm{v} / \mathrm{v})$ of exponentially growing precultures. The pre-cultures of $E$. coli $\mathrm{K} 12$ were grown in $12.5 \mathrm{~mL}$ Luria-Bertani complex medium containing 5 g/L yeast extract, $10 \mathrm{~g} / \mathrm{L}$ tryptone and $5 \mathrm{~g} / \mathrm{L}$ sodium chloride. $H$. polymorpha was pre-cultivated in yeast extract (10 g/L)/peptone (20 g/L) complex medium at $\mathrm{pH} 6.5$ containing $20 \mathrm{~g} / \mathrm{L}$ glucose [38,40]. All pre-cultures were inoculated with $1 \mathrm{~mL}$ glycerol stock (750 $\mu \mathrm{L}$ culture grown on respective complex medium $+250 \mu \mathrm{L} 80 \%$ (w/w) glycerol).

\subsection{Refining the Design of Two Small-Scale Bioreactors Using Autoclavable Glass Bonding}

\subsubsection{Bonded RAMOS Flask}

The Respiration Activity MOnitioring System (RAMOS) allows the online monitoring of respiration activity as the key variable in shake flasks. The respiration rates for oxygen and carbon dioxide are the most suitable measur- 
able variables to quantify the physiological state of aerobic microorganisms. The current RAMOS flasks are produced by melting threads to a conventional $250 \mathrm{~mL} \mathrm{Er-}$ lenmeyer flask (Figure 2(a)). The newly designed bonded RAMOS flask (Figure 2(b)) consists of a stumped flask covered by a borosilicate glass plate bonded on top. The stumped flask was produced from a commercial $250 \mathrm{~mL}$ Erlenmeyer flask that was cut off at a height of $52 \mathrm{~mm}$ using a diamond cutter (Quarzglas Heinrich, Aachen/ Germany). The cover plate was equipped with four borosilicate glass connection threads for aeration (GL 18), sensor fixation (GL 32), and sampling (GL 18) (all from Duran, Mainz/Germany). The holes in the cover plate for the threads were drilled with a diamond drill (Quarzglas Heinrich, Aachen/Germany). Furthermore, two aeration tubes in the flask were made of stainless steel 1.4301 (mechanical workshop, AVT. BioVT, RWTH Aachen University). All cut faces were smoothed with corundum 400.

Since it is known that the side of float glass that has been in contact with the tin bath during the production process (referred to as the "tin side") has altered surface

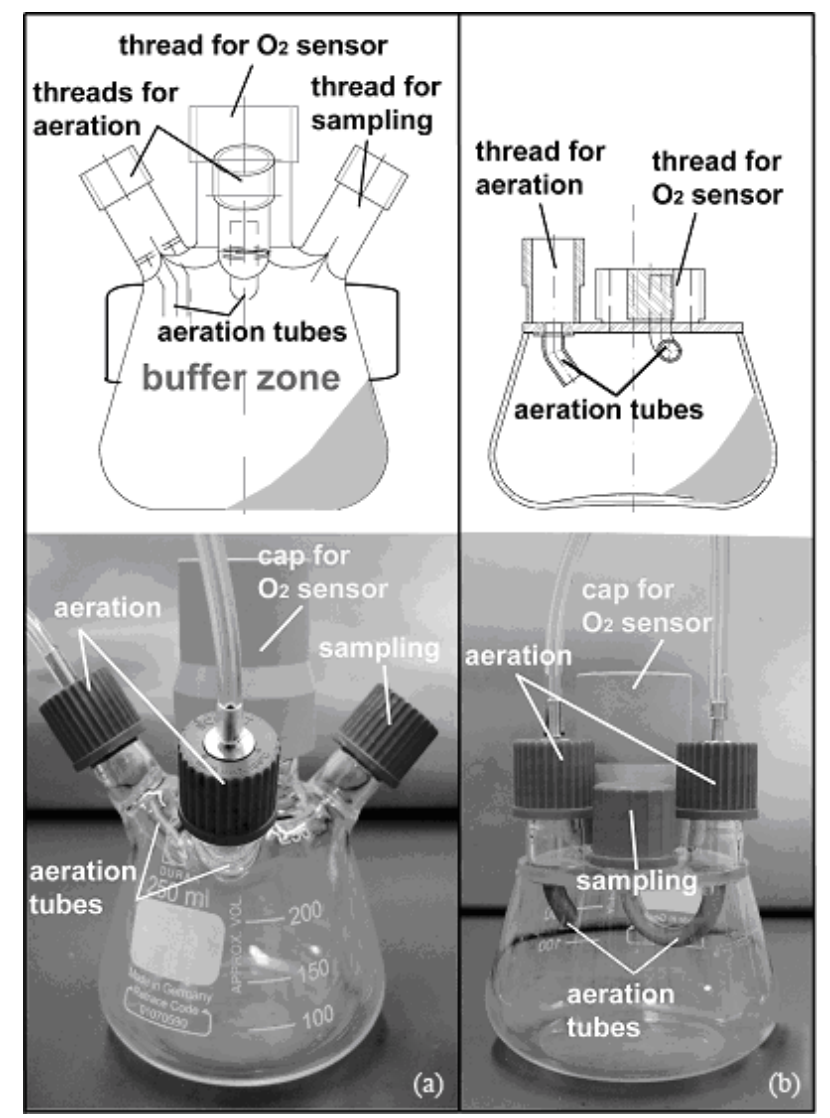

Figure 2. Design of the original melted-joined RAMOS flask (a) and the newly designed bonded one (b); new RAMOS flask bonded with GB 368 (Delo, 15 s cured using UV light (366 $\mathrm{nm})$ ), joint faces smoothed with corundum 400 and degreased with 2-butanone. properties [41,42], it may affect the durability of adhesive bonds. This side of the cover plate was identified using UV light at $254 \mathrm{~nm}$ (UV lamp NU 4 watt; Roth, Darmstadt/Germany). The tin ions fluoresce and produce a milky white image that is slightly visible to the human eye in a darkened room [43]. The tin side was determined as the bottom side of the flask bonded onto the flask.

Before application of the urethane acrylate adhesive GB 368, all joint faces were degreased with MEK. The curing was performed using a UV lamp NU 4 watt at $366 \mathrm{~nm}$ (Roth, Darmstadt/Germany) according to the manufacturer's specifications. The adhesive thickness was controlled at $0.2 \mathrm{~mm}$ by glass beads (Sigmund Lindner $\mathrm{GmbH}$, Warmensteinach/Germany). First, the aeration tubes were bonded on the bottom side of the cover plate and then the threads were bonded on the upper side. After curing of $24 \mathrm{~h}$, the so equipped cover plate was then bonded onto the flask stump.

\subsubsection{Validation of the Newly Bonded RAMOS Flask}

First, the tight fitting of the aforementioned bonds was checked, whereby the inner pressure of the flasks was increased by 25 mbar and monitored in the RAMOS device for $16.5 \mathrm{~h}$. The maximum oxygen transfer capacity of the newly designed RAMOS flask was determined based on sulfite oxidation according to Hermann et al. [44] and Maier et al. [3]. The measurements were performed at $37^{\circ} \mathrm{C}$ in a RAMOS device with a shaking frequency of $350 \mathrm{rpm}$ and a shaking diameter of $50 \mathrm{~mm}$. The filling volume was varied from 10 to $15 \mathrm{~mL}$.

To compare the conventionally melted RAMOS flasks and the newly bonded RAMOS flasks in biological experiments, parallel cultivations of the model organisms $E$. coli K12 (DSM 498) and the yeast $H$. polymorpha wild type strain (DSM 70277) were performed in the RAMOS device as described in Section 2.4.4.

\subsubsection{New Design of COSBIOS Reactor}

The Continuous Operated Shaken BIOreactor System (COSBIOS) enables parallel continuous fermentations on a small scale [45]. Figure 3 compares the design and fabrication of the current clamped COSBIOS reactor to that of the newly designed bonded reactor. The newly designed COSBIOS reactor (Figure 3(b)) consists of a borosilicate glass cylinder, an outlet, a screw cap, a bottom plate, and a threaded ring. All of the last three components consist of stainless steel 1.4301. The screw cap is made of poly-ether-ether-ketone. Furthermore, the cover plate (Quarzglas Heinrich, Aachen/Germany) is equipped with five GL 14 threads (Duran, Mainz/Germany). A hole was tangentially drilled into the glass cylinder (Quarzglass Heinrich, Aachen/Germany). The steel outlet was fit to the outer cylinder shape and bonded on 
the hole (Figure 3(b)). Afterwards, the cylinder was bonded into a notch of the bottom stainless steel plate. Finally, the threaded ring below the screw cap was bonded to the top of the glass cylinder. GB 368 was used for all bonds. Curing was performed according to Section 2.5.1.

\section{RESULTS AND DISCUSSION}

\subsection{Investigation of Practice-Relevant Parameters}

The lifespan of the bonds [18] is not only influenced by the composition of the adhesive and materials but also by the application conditions. Therefore, the practice-relevant parameters of cleaning and fermentation compounds as well as the effect of adhesive on the actual cultivated microorganisms were investigated in this study.

\subsubsection{Cleaning}

One of the most important factors affecting the adhesive joints and respective bonded glass devices is multiple cleaning. Thereby, the cleaning process can affect the adhesive or the glass substrate: The cleaning agents can penetrate into the polymer matrix and sharp temperature changes can stress the adhesive polymer. Furthermore, alkaline cleaning agents can cause glass corrosion. In general, cleaning material is longer exposed to high temperatures (up to $85^{\circ} \mathrm{C}$ ) in SM1 than in SM2 which may thermally stress the adhesive bonds. Further load

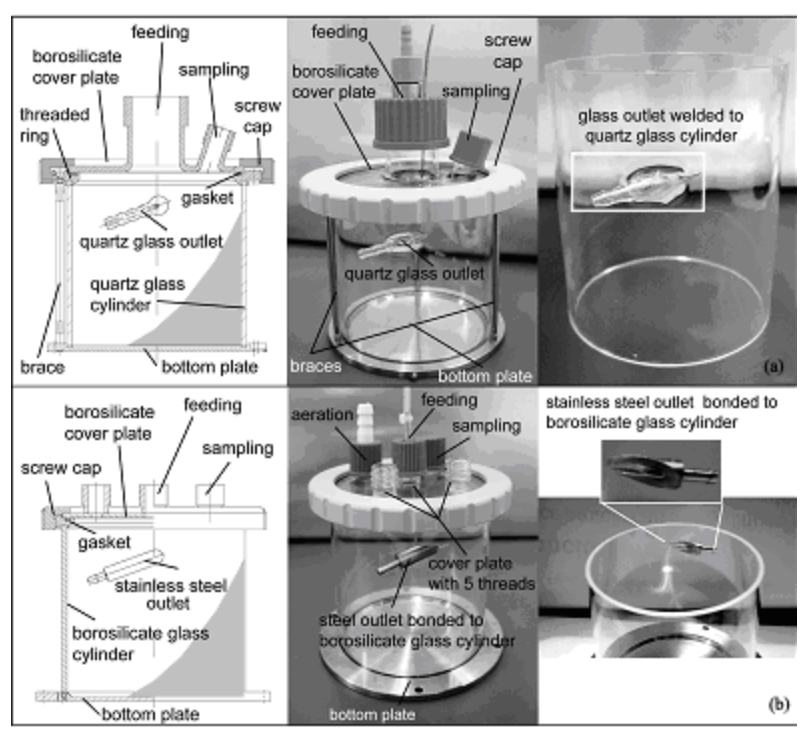

Figure 3. Comparison of the original COSBIOS reactor (a) and the reactor refined using autoclavable glass bonding (b). The original reactor consists of a quartz glass outlet welded to a quartz glass cylinder while the refined COSBIOS reactor consists of a stainless steel outlet bonded to a borosilicate glass cylinder. Bonding with GB 368 (Delo, cured 15 s using UV light (366 nm)) after degreasing with 2-butanone. are frequent and rapid temperature changes as well as the additional alkaline cleaning phase. Cleaning programs for medical devices such as SM2 commonly contain two alkaline cleaning steps: one to remove organic debris and one for disinfection (for SM2, at high temperature of $93^{\circ} \mathrm{C}$ ). For disinfection, hydrogen peroxide is used which can also attack the adhesive joint and the glass substrate as well.

For the substrate combination bor-bor, the shear strength of specimens bonded with the urethane acrylate GB 368 (Figure 4(a)) and degreased with MEK decreased from 13.4 MPa to 7.6 MPa after cleaning in SM1 and decreased from 13.4 MPa to 11.0 MPa after
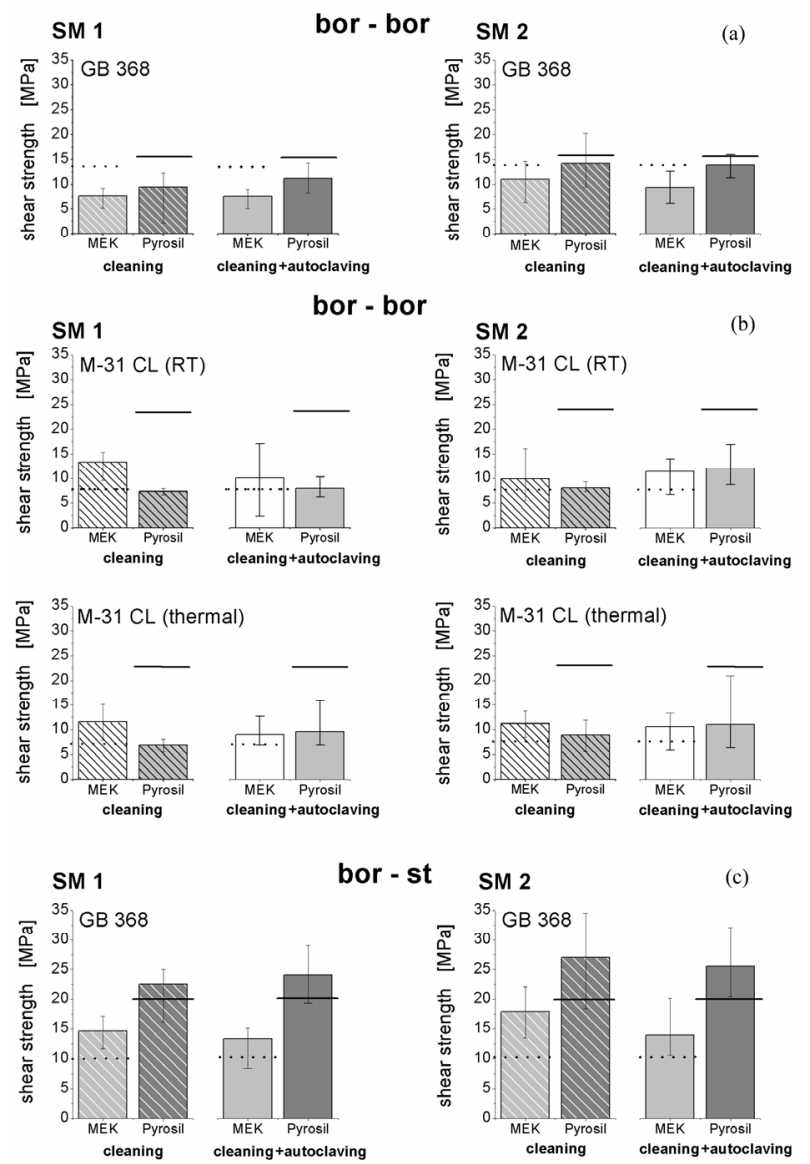

Figure 4. Influence of cleaning and additional autoclaving on shear strength of borosilicate glass/borosilicate glass and borosilicate glass/stainless steel bonds. Specimens: borosilicate glass bonded to itself (bor-bor) or to stainless steel (bor-st) (Figure 1); urethane acrylate adhesive Delo GB 368 was compared to two-component epoxy Henkel-Loctite M-31 CL; pre-treatment consisted of degreasing with 2-butanone (MEK) or additionally treated with Pyrosil ${ }^{\circledR}$; shear strength after 50 cleaning cycles (SM1 and SM2, striped bars) and additional 50 autoclaving cycles $\left(20 \mathrm{~min}\right.$ at $121^{\circ} \mathrm{C}$ and 2 bar, solid-colored bars) in comparison to shear strength of respective unaged specimens degreased with MEK (black dotted line) or additionally pre-treated with Pyrosil ${ }^{\circledR}$ (black line), respectively. 
cleaning in SM2. A similar trend occurred after 50 additional autoclaving cycles, whereby the shear strength changed from 13.4 MPa to 9.4 MPa and to 14.2 MPa for the respective SM1 and SM2 cleaning programs. During the subsequent autoclaving, the shear strength increased almost up to those values of the unaged specimens. Specimens additionally pre-treated with Pyrosil ${ }^{\circledR}$ showed higher shear strengths both before (16.3 MPa) and after the two aging steps (12.7 MPa in SM1 and 13.9 MPa in SM2) where no post-curing occurred. Thereby, a lower loss of shear strength was observed for Pyrosil ${ }^{\circledR}$-treated samples than the loss of shear strength of only degreased specimens, in particular for GB 368 specimens cleaned in SM2. Consequently, multiple cleaning, both in SM1 and SM2, did not significantly affect the bond durability of the urethane acrylate GB 368. The shear strength loss is lower for cleaning in SM2 indicating that longer exposure to high temperatures and rapid temperature changes of SM1 affect the bond durability more significantly.

By contrast, the specimens bonded with the twocomponent epoxy M-31 CL (Figure 4(b)) and additionally pre-treated with Pyrosil ${ }^{\circledR}$ lose one-half to two-thirds of their initial shear strength of $23 \mathrm{MPa}$ due to aging during both cleaning programs. Nevertheless, the shear strengths increased during autoclaving, which implies a post-curing by the steam sterilization. Interestingly, the shear strength of specimens degreased with MEK increased after cleaning in both programs as well as after additional autoclaving compared to the initial shear strength tested before treatment. On the other hand, the initial shear strength of those specimens degreased with MEK was extremely low (7.8 $\mathrm{MPa}$ ) compared to those of Pyrosil ${ }^{\circledR}$ pre-treated specimens (23.2 MPa). Moreover, glass breakage was observed as a material failure, whereby the adhesive bonds stay intact independent of treatment. This infers that cleaning does not significantly weaken the adhesive joints.

Concerning the substrate combination bor-st (Figure 4(c)), the shear strength of the urethane acrylate adhesive GB 368 increased after cleaning in SM1 and SM2 for both applied pre-treatments. After autoclaving, the respective shear strengths further increased. Here, a significant post-curing of GB 368 occurred. The observed failure modes shifted from glass breakage before cleaning to adhesive fractures on the glass side after cleaning. After steam sterilization, adhesive fractures were also observed at the stainless steel surface as well. Therefore, cleaning as well as autoclaving harmed the adhesive joints of bor-st substrates although the absolute shear strength increased during the accelerated aging. Moreover, the actual attack of the cleaning process and the autoclaving process differ. Whereas cleaning corrodes the glass, the steam sterilization corrodes the steel surface, which subsequently catalyzes the polymer degradation of the adhesive [46].

Comparing the shear strengths of bor-bor and bor-st specimens, the shear strength of the bor-bor specimens decreased. However, the shear strength of bor-st samples rose significantly indicating a post-curing. The results concerning the cleaning programs SM1 and SM2 did not differ. To compare the two curing cycles (at ambient temperature and at $65^{\circ} \mathrm{C}$, respectively) of the epoxy resin M-31 CL, the residual shear strengths were similar both after cleaning and autoclaving. Thus, the thermal curing did not lead to improved lifespan as has been already observed in a previous study [18].

In summary, cleaning — even in worst-case scenariosdid not adversely affect the shear strength of adhesive joints. Nevertheless, the tested duration of cleaning was rather short with respect to long-term lifespan of adhesive joints in autoclaving (i.e. 800 cycles). It can be expected that multiple cleaning steps will harm the bond itself and hence shorten the lifespan of a bioreactor. In general, it can be concluded that both GB 368 and M-31 CL adhesives are suited for application in biotechnology and medicine. Regarding the substrate combination bor-bor, both tested adhesives behaved similarly during the cleaning procedures in SM1 and SM2. Moreover, the thermal curing had no effect on the M-31 CL bonds. In the bor-st joints, however, the bond shear strength increased during cleaning and, in particular, during the subsequent autoclaving. In this case, differences in shear strength after cleaning in SM1 and SM2 were also observed.

\subsubsection{Loading during Autoclaving}

The bond durability under load during autoclaving was investigated. Thus, the adhesives tested in a previous study showed only very low shear strength under autoclaving conditions due to softening of the adhesive polymer and, subsequently, resulting in a reduced capability to bear loads [18]. Therefore, bor-bor and bor-st specimens were loaded during the autoclaving process with $143 \mathrm{~g}$, which is the weight of a fully equipped RAMOS flask as one of the reactors refined in this study. All bor-bor specimens already failed in the first 11 autoclaving cycles, whereas the single lap joints of the bor-st substrate combination withstood 100 autoclaving cycles (data not shown). Interestingly, the shear strength loss of the latter combination was significantly higher in the previous study (50\% instead of $18 \%$ ). However, this accelerated failure of the bor-bor specimens can be explained by the shear stress of the bor-bor specimens applied at an angle $90^{\circ}$ of the unsupported adherent (Figure 1). By contrast, the joint face of the bor-st specimens was compressively loaded instead of by shear 
stress and the load can be borne by the second adherent (Figure 1). Consequently, shear loading of unsupported adherents - as in the case of the bor-bor specimensshould be avoided.

\subsubsection{Fermentation Compounds}

The cultivation medium and metabolic products come into contact with the adhesive and, consequently, can influence it. In this regard, ethanol and acetic acid, both being either potential substrates or products in bioprocesses, were examined in the presence of adhesive bonds.

HPLC measurements showed that the ethanol concentration decreased from $120 \mathrm{~g} / \mathrm{L}$ to $3.76 \mathrm{~g} / \mathrm{L}$ during 10 autoclaving cycles because ethanol is highly volatile. The acetic acid concentrations only slightly dropped from $200 \mathrm{~g} / \mathrm{L}$ to $192.5 \mathrm{~g} / \mathrm{L}$.

After 25 and 50 autoclaving cycles, the shear strength of the respective adhesive joints dramatically decreased (Figure 5). Thus, the shear strength of bor-bor specimens, degreased with MEK declined from 13.4 MPa before aging to $7.9 \mathrm{MPa}$ after 25 autoclaving cycles and to 7.0 MPa after 50 autoclaving cycles. A similar trend was observed for Pyrosil ${ }^{\circledR}$ treated bor-bor samples (6.6 MPa after 25 and 50 cycles, respectively).

For the substrate combination bor-st, the shear strength loss was more dramatic. Here, the shear strength of previously degreased specimens (MEK) dropped from 19.9 MPa to 6.4 MPa after 25 autoclaving cycles and to 2.1 MPa after 50 autoclaving cycles meaning, inferring a shear strength loss of up to $90 \%$. Compared to the shear strength loss of the joints observed after 400 standard cycles of steam sterilization without ethanol or acetic acid, the loss observed in the presence of those fermentation compounds was more than $30 \%$ higher [18]. The
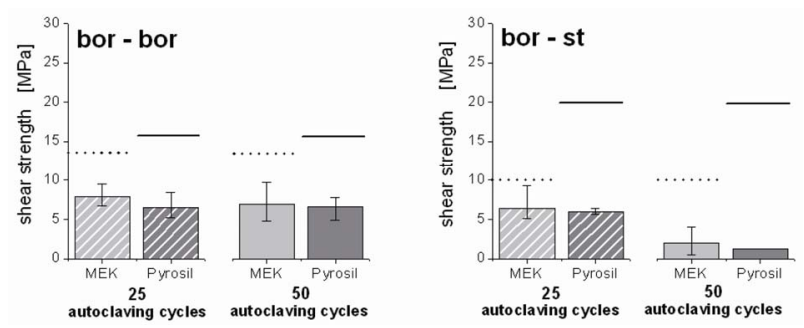

Figure 5. Influence of the fermentation compounds ethanol and acetic acid on adhesive joints for the substrate combinations borosilicate glass bonded to itself (bor-bor) or to stainless steel (bor-st) using the urethane acrylate Delo GB 368 with the two pre-treatments of 2-butanone degreasing (MEK) and additional Pyrosil ${ }^{\circledR}$ treatment; shear strength after 25 (striped bars) and 50 (solid bars) autoclaving cycles $\left(20 \mathrm{~min}\right.$ at $121^{\circ} \mathrm{C}$ and 2 bar) in a solution of $15 \%(\mathrm{w} / \mathrm{v})$ ethanol and $20 \%(\mathrm{w} / \mathrm{v})$ acetic acid; the shear strength of all the autoclaved specimens were compared to those of unaged specimens pre-treated with MEK degreasing (black dotted line) and additional Pyrosil ${ }^{\circledR}$ pretreatment (black line).
Pyrosil $^{\circledR}$ treated samples already failed after 50 autoclaving cycles. This development indicates a dramatic attack on the adhesive joints by ethanol and acetic acid. Since high concentrations of these common fermentation compounds lead to that dramatic boost of adhesive joint aging, it can be concluded that other reactive fermentation compounds also adversely affect the bond durability. Therefore, the contact between fermentation compounds and the joint faces should be avoided in the reactor design, if possible.

The shear strength loss for the respective bor-st joints was much more pronounced than that of bor-bor specimens. After 50 autoclaving cycles, the bor-st bonds totally failed. This failure can be attributed to steel corrosion. An additional pre-treatment of the stainless steel substrate could prevent this corrosion. In this context, the Pyrosil ${ }^{\circledR}$ pre-treatment, which physicochemically modifies the surface of stainless steel, did not lead to an improved bond lifespan, although it forms durable joints that withstand frequent autoclaving in the absence of ethanol and acetic acid. When the aggressive compound acetic acid is present, the failure may be attributed to reaction of acetic acid with e.g. the siloxane groups of borosilicate glass formed by the Pyrosil ${ }^{\circledR}$ pre-treatment. Furthermore, this may explain the generally lower residual shear strength of Pyrosil ${ }^{\circledR}$-treated specimens-independent whether bor-bor or bor-st was applied.

\subsubsection{Interactions of Adhesives and Cultivated Microorganisms}

If microorganisms come into contact with the adhesives, they can metabolize the respective adhesive or their metabolic products can react with the adhesive. To investigate these possible microbial effects, the bacterium $E$. coli $\mathrm{K} 12$ and the yeast $H$. polymorpha wild type strain were cultivated in the presence of adhesive disks.

Some of the investigated adhesive disks, e.g. the (urethane) acrylates GB 310 and GB 368 and Vitralit 6128, became opaque due to autoclaving during the cultivation processes. Moreover, transparent adhesives discolored during the cultivation itself and/or during the following autoclaving. The degree of discoloring correlates well with the pigmentation of the cultivated microorganisms and the medium. Since the yeast $H$. polymorpha and its cultivation medium are pigmented more intensively, the adhesive disks cultivated in the respective batches showed a more intense color. Nevertheless, other changes in the adhesive disk surface such as roughening were not observed.

The respective RAMOS curves exemplarily show the same progress for E. coli K12 cultures (Figure 6(a)) in the presence of the acrylate adhesive Vitralit 7563 and the two-component epoxy adhesive EPVP 4611/4 compared to the growth without any adhesive (control batch). 
In addition, no differences in the respiration activity of the yeast H. polymorpha (Figure 6(b)) were observed in the presence of the (urethane) acrylates GB 310 and GB 368. Therefore, the tested adhesives did not affect the microbial growth of the chosen model organisms.

\subsection{Refining the Design of Two Small-Scale Bioreactors Using the Autoclavable Glass Bonding}

Based on previous work [18] and the aforementioned results, the design of two glass bioreactors for smallscale fermentations was refined using the newly developed autoclavable glass bonding technique. The basis for the design optimization was a conventional RAMOS (Respiration Activity MOnitoring System) flask with melted bor-bor joints. Moreover, as a second example, a typical COSBIOS (Continous Operated Shaken BIOreactor System) reactor with clamped glass steel joints served as the starting point for the design refinement.

\subsubsection{Bonded RAMOS Flask}

To produce the original RAMOS flask, threads for aeration, sensor fixation and sampling were melted onto a conventional $250 \mathrm{~mL}$ Erlenmeyer flask (Figure 2(a)). These modifications by melting inherently lead to a poor geometric reproducibility of the top part of the flasks due to the large deformed area by the thermal treatment. To guarantee identical geometries and, therefore, fluiddynamic conditions in the bottom part of the flasks as in a conventional shake flask, this RAMOS flask has a "buffer zone" between the upper part and the bottom part (Figure 2(b)), defined by the maximal height of the rotating fluid. The buffer zone results in an enlarged head-
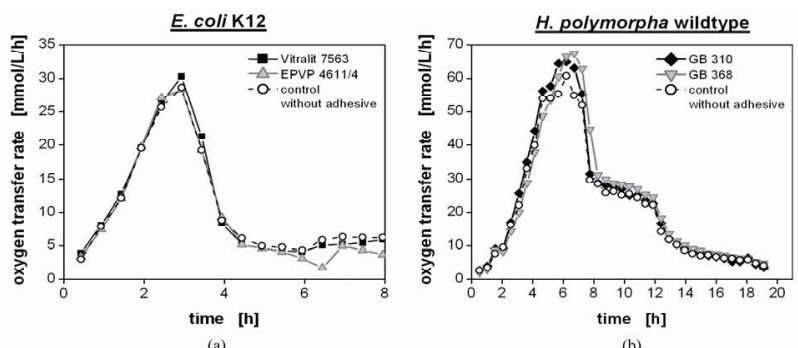

(b)
Figure 6. Investigation of potential influence of the adhesives on microbial activity as exemplarily shown in cultivations of model organisms Escherichia coli K12 (a) and yeast Hansenula polymorpha wild type (b); for convenience, each curve represents identical technical triplets; control batches without any adhesive represented by open circles. Cultivation of EScherichia coli K12: In the presence of the acrylate Vitralit 7563 (square, Panacol-Elosol, cured by UV light, $15 \mathrm{~s}$ ) and the twocomponent epoxy EPVP 4611/4 (triangle, Polytec PT, cured $16 \mathrm{~h}$ at RT); Cultivation of Hansenula polymorpha wild type: In the presence of the acrylates GB 310 (diamond, Delo, cured by UV light, 20 s) and GB 368 (inverse triangle, Delo, cured by UV light, $10 \mathrm{~s})$. space volume, reducing the sensitivity of the measuring device.

The design of the RAMOS flask was refined, whereby a conventional $250 \mathrm{~mL}$ Erlenmeyer flask was cut down to a height of $52 \mathrm{~mm}$, corresponding to operation conditions as $400 \mathrm{rpm}$ shaking frequency, a $15 \mathrm{~mL}$ filling volume, and a $50 \mathrm{~mm}$ shaking diameter. Consequently, the buffer zone could be eliminated (Figure 2(b)), thereby increasing the geometric reproducibility and the measuring sensitivity. On this $52 \mathrm{~mm}$ high stump, a cover plate made of borosilicate glass and equipped with connections for aeration, sensor fixation and sampling was bonded. Figure 2 illustrates the comparison of the newly designed RAMOS flask to the conventional joint-melted one. Seven of these bonded prototypes were fabricated.

The geometric reproducibility of the bonded RAMOS flasks was compared to that of the conventional flasks. The average volume of the seven bonded prototypes was $228.3 \mathrm{~mL}$ versus $278.5 \mathrm{~mL}$ of the conventional RAMOS flasks (56 flasks), which means a volume reduction of $18 \%$. Furthermore, the volume of the bonded flasks deviated maximally $2 \mathrm{~mL}$ from the mean value which is within the production tolerance of conventional $250 \mathrm{~mL}$ Erlenmeyer flasks; this implies an enhanced geometric reproducibility of the newly designed bonded flasks.

Since these reactors are utilized to monitor the oxygen transfer rate (OTR), the carbon dioxide transfer rate (CTR) and the respiratory quotient (RQ) by an exhaust gas analysis, the tightness of flasks is essential to obtain accurate results. During $16.5 \mathrm{~h}$, the pressure losses in bonded RAMOS flasks amounted $0.0108 \mathrm{mbar} / \mathrm{h}$ compared to $0.094 \mathrm{mbar} / \mathrm{h}$ in melted-joined flasks (data not shown). Therefore, the bonded RAMOS flasks showed a similar tight fitting as the melted-joined ones and can be applied to RAMOS measurements. Moreover, the maximum oxygen transfer capacity (OTRmax) of the newly designed RAMOS flask was determined as $45 \mathrm{mmol} / \mathrm{L} / \mathrm{h}$ for a filling volume of $10 \mathrm{~mL}$ and as $37 \mathrm{mmol} / \mathrm{L} / \mathrm{h}$ for $15 \mathrm{~mL}$. Similar values were obtained in the melted-joined flasks with $52 \mathrm{mmol} / \mathrm{L} / \mathrm{h}$ for a filling volume of $10 \mathrm{~mL}$ and 37 $\mathrm{mmol} / \mathrm{L} / \mathrm{h}$ for a filling volume of $15 \mathrm{~mL}$.

The bonded flasks were evaluated in various cultivation experiments by comparison to the joint-melted flasks. Cultivations of the aforementioned model organisms $E$. coli $\mathrm{K} 12$ and $H$. polymorpha wild type showed no differences between both types of RAMOS flasks, as can be seen from Figure 7. Consequently, the newly bonded RAMOS flasks with a higher geometric reproducibility can indeed replace the conventional RAMOS flasks. The adhesively bonded prototypes have been routinely used in the laboratory and already have withstood quently, the newly bonded RAMOS flasks with a higher geometric reproducibility can indeed replace the con- 

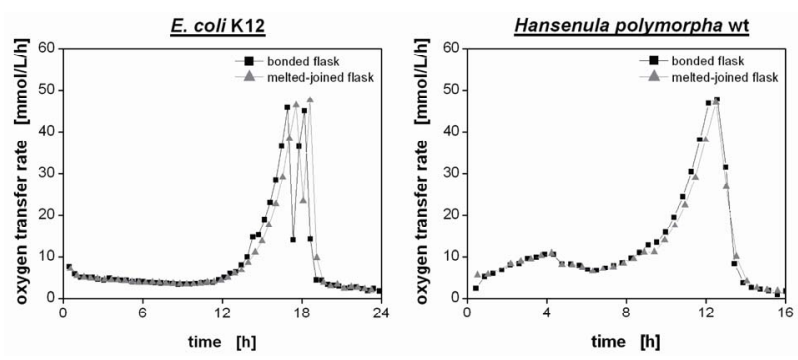

Figure 7. Evaluation of melted-joined and bonded RAMOS flasks in cultivations of model organisms Escherichia coli K12 and yeast Hansenula polymorpha wild type. Cultivation of $E$. coli K12: shake flask culture in $10 \mathrm{~mL}$ Wilms-MOPS medium (initial $\mathrm{pH} 7.5$ ) at $37^{\circ} \mathrm{C}$ with a shaking frequency of $350 \mathrm{rpm}$ and a shaking diameter of $50 \mathrm{~mm}$; Cultivation of $H$. polymorpha wild type: shake flask culture in $10 \mathrm{~mL}$ Syn6-MES medium (initial $\mathrm{pH}$ 6.4) at $37^{\circ} \mathrm{C}$ with a shaking frequency of $350 \mathrm{rpm}$ and a shaking diameter of $50 \mathrm{~mm}$.

ventional RAMOS flasks. The adhesively bonded prototypes have been routinely used in the laboratory and already have withstood more than 100 autoclaving cycles.

\subsubsection{New Design of the COSBIOS Reactor}

The COSBIOS reactor is used as a small-scale reactor for parallel continuous fermentations. Due to time-consuming operation mode, such fermentations are costly and rarely performed with conventional equipment [45,47]. The small scale COSBIOS combines the advantages of shaken bioreactors with the advantages of continuous fermentation [47]. Because of its small scale and its simple handling, it is possible to operate many of these shaken bioreactor vessels simultaneously. By using parallel vessels, biological cultures can be characterized in an efficient manner, thus saving money, materials, and time [45]. Since the reactor cylinder is made of expensive quartz glass, it has been selected as a model reactor to refine bioreactor design using autoclavable glass bonding.

A conventional COSBIOS reactor (Figure 3(a)) consists of a quartz glass cylinder to which a glass outlet is tangentially welded. Afterwards, a connection hole is drilled through the outlet (Figure 3(a), right side). The aforementioned procedure can only be realized by using quartz glass, because thermal treatment leads to the softening of a small and very defined area only, in opposite to borosilicate glass. However, quartz glass is very costly. The cylinder is clamped onto a two-component bottom plate and a threaded ring made of stainless steel. A cover plate made of borosilicate glass and equipped with various borosilicate glass threads for feeding and sampling is fixed on a threaded ring using a screw cap made of polyether-ether-ketone. Due to the clamping, the reactor handling is very complicated and time-consuming. Moreover, the risk of contamination and stress ruptures of the costly quartz glass cylinder is increased, in particular, by the significant differences between the thermal expansions of quartz glass $\left(1 \times 10^{-6} \mathrm{~K}^{-1}\right)$ and stainless steel $(17$ $\times 10^{-6} \mathrm{~K}^{-1}$ ).

Figure 3 compares this conventional COSBIOS reactor to the newly designed one, which is assembled using autoclavable glass bonding. The bonded reactor (Figure 3(b)) consists of a stainless steel outlet instead of a glass one. The stainless steel outlet is bonded to the borosilicate glass cylinder (Figure 3(b), right side). Since this outlet does not have to be welded to the cylinder, the quartz glass cylinder can be replaced by less expensive borosilicate glass material. The use of a stainless steel outlet guarantees an enhanced geometric reproducibility of this component compared to that made of quartz glass, because it can be produced with higher precision. Unlike the conventional COSBIOS reactor, the cylinder was directly bonded onto the bottom plate, which can now be considered as a single-part component. Hence, clamping is no longer necessary and the former three gaskets could be replaced by simply one (between cover plate and the screw cap).

To guarantee a high application flexibility of the bonded reactor and easy cleaning, the cover plate was not bonded to the cylinder but is still fixed by a polyether-ether-ketone screw cap. Nevertheless, the number of components was reduced from fourteen to six due to the omission of braces, thus simplifying the design and handling of this new bioreactor. Furthermore, the diameter of the threaded ring and the screw cap could be minimized, thereby saving material, in particular the extremely expensive poly-ether-ether-ketone, and weight.

To summarize the refinements of the COSBIOS reactor achieved using autoclavable glass bonding results in the following advantages:

- The production process is simplified due to omission of braces and drill holes.

- Material is saved due to the minimized diameter of the screw cap and threaded ring.

- Costs are reduced by switching to less expensive borosilicate glass and by reducing the amount of poly-ether-ether-ketone needed.

- The handling is simplified due to reduction of components (six instead of fourteen).

- The weight of reactor was diminished because of less material needed and which minimizing the risk of shaker imbalances, thereby prolonging the lifespan of expensive shakers.

- The risk of stress ruptures is minimized and finally resulting in an improved lifespan of the reactors and, subsequently, in a further cost reduction.

- The risk of contamination is minimized, thereby 
enhancing profitability of elaborate and time-consuming continuous fermentations.

These numerous advantages illustrate the high potential of autoclavable glass bonding to simplify fabrication and handling as well as the profitability of perspective bioreactors.

\section{CONCLUSIONS}

Glass bonding greatly enhances the geometric reproducibility of bioreactors. As it is easy to handle, it opens up new opportunities to design bioreactors that had been previously too expensive and complicated. Thus, by offering more degrees of freedom, these autoclavable glass bonding techniques ultimately break down current design barriers. Designs can be implemented which have been impossible so far because of the lack of a suited technology.

\section{ACKNOWLEDGEMENTS}

This research project was funded by the German Federal Ministry of Economics and Technology in Berlin via the German Federation of Industrial Research Associations "Otto von Guericke” e.V. (AiF) in Cologne (AiF $14487 \mathrm{~N}$ ) and was supported by the research association of DECHEMA e.V. (the Society for Chemical Engineering and Biotechnology). The authors are especially grateful to Regina Thiele for her help and advice. Furthermore, the authors wish to thank the industrial partners who collaborated in this project. In particular, we would like to express our special thanks to Miele \& Cie. KG for performing the cleaning trials. The glass materials were kindly provided by Duran Group GmbH as well as the stainless steel specimens by the LKT $\mathrm{GmbH}$.

\section{REFERENCES}

[1] Büchs, J. (2001) Introduction to advantages and problems of shaken cultures. Biochemical Engineering Journal, 7, 91-99. doi:10.1016/S1369-703X(00)00106-6

[2] Maier, U. and Büchs, J. (2001) Characterisation of the gas-liquid mass transfer in shaken bioreactors. Biochemical Engineering Journal, 7, 99-106. doi:10.1016/S1369-703X(00)00107-8

[3] Maier, U., Losen, M. and Büchs, J. (2004) Advances in understanding and modeling the gas-liquid mass transfer in shake flasks. Biochemical Engineering Journal, 17, 155-167. doi:10.1016/S1369-703X(03)00174-8

[4] Galindo, E., Flores, C., Larralde-Coronab, P., CorkidiBlanco, G., Rocha-Valadez, A.J. and Serrano-Carreón, L. (2004) Production of 6-pentyl- $\alpha$-pyrone by Trichoderma harzianum cultured in unbaffled and baffled shake flasks. Biochemical Engineering Journal, 18, 1-8. doi:10.1016/S1369-703X(03)00115-3

[5] Calam, C.T. (1996) Shake flask fermentations. In: Demain, A.L. and Solomon, N.A. Eds., Manual of Industrial Microbiology and Biotechnology, American Society for Microbiology, Washington, DC, 59-65.

[6] Anderlei, T., Zang, W., Papaspyrou, M. and Büchs, J.
(2004) Online respiration activity measurement (OTR, CTR, RQ) in shaken flasks. Biochemical Engineering Journal, 17, 187-194. doi:10.1016/S1369-703X(03)00181-5

[7] Peter, C.P., Lotter, S., Maier, U. and Büchs, J. (2004) Impact of out-of-phase conditions on screening results in shaking flask experiments. Biochemical Engineering Journal, 17, 205-215. doi:10.1016/S1369-703X(03)00179-7

[8] Losen, M., Frölich, B., Pohl, M. and Büchs, J. (2004) Effect of oxygen limitation and medium composition on Escherichia coli fermentation in shake-flask cultures. Biotechnology Progress, 20, 1062-1068. doi:10.1021/bp034282t

[9] Gaden, E.L. (1962) Improved shaken flask performance. Biotechnology and Bioengineering, 4, 99-103. doi:10.1002/bit.260040112

[10] McDaniel, L.E., Bailey, E.G. and Zimmerli, A. (1965) Effect of oxygen supply rates on growth of Escherichia coli. Part I. Studies in unbaffled and baffled shake flasks. Applied and Environmental Microbiology, 13, 109-114.

[11] Delgado, G., Topete, M. and Galindo, E. (1989) Interaction of cultural conditions and end-product distribution in Bacillus subtilis grown in shake flasks. Applied Microbiology and Biotechnology, 31, 288-292. doi:10.1007/BF00258411

[12] Moore, J.H., Davis, C.C. and Coplan, M.A. (1991) Building scientific apparatus-A practical guide to design and construction. Perseus Books, Reading, Masachusettes, 55-58.

[13] Storhas, W. (1994) Bioreaktoren und periphere Enrichtungen. Vieweg Verlag, Braunschweig/Wiesbaden, 165-166. doi:10.1007/978-3-642-57973-8

[14] Krahe, M. (2007) Biochemical engineering. Ullmann's Biotechnology and Biochemical Engineering, 1st Edition, Wiley-VCH, Weinheim, 2, 655.

[15] Adams, R.D., Comyn, J. and Wake, W.C. (1997) Structural adhesives joints in engineering. 2nd Edition, Chapamn \& Hall, London.

[16] Gleich, D.M., Van Tooren, M.J.L. and Beukers, A. (2002) Structural bonded joint analysis: An overview. In: Mittal, K.L. Ed., Adhesive Joints: Formation, Characteristics and Testing, 2, VSP BV, Utrecht, 159-199.

[17] Pocius, A.V. (2002) Adhesion and adhesives technologyAn introduction. 2nd Edition, Hanser, München, 2-5.

[18] Bähr, C., Stammen, E., Thiele, R., Böhm, S., Dilger, K. and Büchs, J. (2011) Withstanding frequent steam sterilisation: Innovative technique to bond glass and stainless steel composites in biotechnology and endoscopic medicine, International Journal of Adhesion and Adhesives, paper unpublished.

[19] Dien, B.S., Cotta, M.A. and Jeffries, T.W. (2003) Bacteria engineered for fuel ethanol production: Current status. Applied Microbiology and Biotechnology, 63, 258-266. doi:10.1007/s00253-003-1444-y

[20] Mielenz, J.R. (2001) Ethanol production from biomass: Technology and commercialization status. Current Opinion in Microbiology, 4, 324-329. doi:10.1016/S1369-5274(00)00211-3

[21] McMillan, J.D. (1997) Bioethanol production: Status and prospects. Renewable Energy, 10, 295-302. doi:10.1016/0960-1481(96)00081-X

[22] Wiegel, J. (1980) Formation of ethanol by bacteria. A 
pledge for the use of extreme thermophilic anaerobic bacteria in industrial ethanol fermentation processes. Cellular and Molecular Life Sciences, 36, 1434-1446. doi:10.1007/BF01960144

[23] Han, K., Lim, H.C. and Hong, J. (1992) Acetic acid formation in Escherichia coli fermentation. Biotechnology and Bioengineering, 39, 663-671. doi:10.1002/bit.260390611

[24] Fukaya, M., Park, Y.S. and Toda, K. (1992) Improvement of acetic acid fermentation by molecular breeding and process development. Journal of Applied Microbiology, 73, 447-454. doi:10.1111/j.1365-2672.1992.tb05004.x

[25] De Ory, I., Romero, L.E. and Cantero, D. (1999) Maximum yield acetic acid fermenter. Bioprocess and Biosystems Engineering, 21, 187-190. doi:10.1007/s004490050661

[26] Park, Y.S., Ohtake, H. and Toda, K. (1990) A kinetic study of acetic acid production by liquid-surface cultures of Acetobacter aceti. Applied Microbiology and Biotechnology, 33, 259-263. doi:10.1007/BF00164518

[27] Devantier, R., Pedersen, S. and Olsson, L. (2005) Characterisation of very high gravity ethanol fermentation of corn mash. Effect of glycoamylase dosage, pre-saccharification and yeast strain. Applied Microbiology and Biotechnology, 68, 622-629. doi:10.1007/s00253-005-1902-9

[28] Fleury, K., Kalina, V. and Zell, J.J. (1997) Vinegar production. European Patent EP 0548552, 15 January 1997.

[29] Salek, A.T. (2002) Classic techniques for improvement of industrial yeast strains. Part I: Construction of ethanol-resistant and osmophilic industrial strains of Saccharomyces cerevisiae by electrofusion. Biotechnologia, 1, 153-174.

[30] Anderlei, T. and Büchs, J. (2001) Device for sterile online measurement of the oxygen transfer rate in shaking flasks. Biochemical Engineering Journal, 7, 157-162. doi:10.1016/S1369-703X(00)00116-9

[31] Rechmann, H., Friedrich, A., Forouzan, D., Barth, S., Schnabl, H., Biselli, M. and Boehm, R. (2007) Characterization of photosynthetically active duckweed (Wolffia australiana) in vitro culture by Respiration Activity Monitoring System (RAMOS). Biotechnology Letters, 29, 971-977. doi:10.1007/s10529-007-9330-9

[32] Jänicke, G., Sauter, C., Bux, R. and Haas, J. (2007) Characterisation of shake flasks for cultivation of animal cell cultures. In: Smith, R. Ed., Cell Technology for Cell Products, Springer, Dordrecht, 727-731.

[33] Peña, C., Peter, C.P., Büchs, J. and Galindo, E. (2007) Evolution of the specific power consumption and oxygen transfer rate in alginate-producing cultures of Azotobacter vinelandii conducted in shake flasks. Biochemical Engineering Journal, 36, 73-80. doi:10.1016/j.bej.2007.02.019

[34] Guez, J.S., Müller, C.H., Danze, P.M., Büchs, J. and Jacques, P. (2008) Respiration activity monitoring system (RAMOS), an efficient tool to study the influence of the oxygen transfer rate on the synthesis of lipopeptide by Bacillus subtilis ATCC6633. Journal of Biotechnology, 134, 121-126. doi:10.1016/j.jbiotec.2008.01.003
[35] Scheidle, M., Jeude, M., Dittrich, B., Denter, S., Kensy, F., Suckow, M., Klee, D. and Büchs, J. (2010) Highthroughput screening of Hansenula polymorpha clones in the batch compared with the controlled-release fed-batch mode on a small scale. FEMS YeastResearch, 10, 83-92. doi:10.1111/j.1567-1364.2009.00586.x

[36] Wilms, B., Hauck, A., Reuss, M., Syldatk, C., Mattes, R., Siemann, M. and Altenbuchner, J. (2001) High-cell-density fermentation for production of L-Ncarbamoylase using an expression system based on the Escherichia coli rhaBAD promoter. Biotechnology and Bioengineering, 73, 95-103. doi:10.1002/bit.1041

[37] Jeude, M. (2007) Entwicklung und anwendung einer fed-batch-betriebsweise mit nährstofffreisetzungssystemen zur kontrollierten kultivierung und zum screening von mikroorganismen in schüttelkolben. Ph.D. Dissertation, RWTH Aachen University, Aachen.

[38] Gellissen, G. (2004) Production of recombinant proteins. Wiley-VCH GmbH, Weinheim, 307-314. doi:10.1002/3527603670

[39] Jeude, M., Dittrich, B., Niederschulte, H., Anderlei, T., Knocke, C., Klee, D. and Büchs, J. (2006) Fed-batch mode in shake flask by slow-release technique. Biotechnology and Bioengineering, 95, 433-436. doi:10.1002/bit.21012

[40] Stöckmann, C., Maier, U., Anderlei, T., Knocke, C., Gellissen, G. and Büchs, J. (2003) The oxygen transfer rate as key parameter of Hansenula polymorpha screening cultures. Journal of Industrial Microbiology and Biotechnology, 30, 613-622. doi:10.1007/s10295-003-0090-9

[41] Le Bourhis, E. (2008) Glass-Mechanics and technology. Wiley-VCH, Weinheim, 208-212.

[42] Frischat, G.H. (2002) Tin ions in float glass cause anomalies. Comptes Rendus Chimie, 5, 759-763. doi:10.1016/S1631-0748(02)01436-4

[43] Yates, B.W. and Duffy, A.M. (2008) Statistical analysis of the metrological properties of float glass. In: Khounsary, A.M., Morawe, C. and Goto, S. Eds., Advances in X-Ray/EUV Optics and Components III, Proceedings of SPIE, 7077, 23 September 2008.

[44] Hermann, R., Lehmann, M. and Büchs, J. (2003) Characterization of gas-liquid mass transfer phenomena in microtiter plates. Biotechnology and Bioengineering, 81, 178-186. doi:10.1002/bit.10456

[45] Akgün, A., Müller, C., Engmann, R. and Büchs, J. (2008) Application of an improved continuous parallel shaken bioreactor system for three microbial model systems. Bioprocess and Biosystems Engineering, 31, 193-205. doi:10.1007/s00449-007-0183-3

[46] Armstrong, K.B. (1997) Long-term durability in water of aluminium alloy adhesive joints bonded with epoxy adhesives. International Journal of Adhesion and Adhesives, 17, 89-105. doi:10.1016/S0143-7496(96)00038-3

[47] Akgün, A., Maier, B., Preis, D., Roth, B., Klingelhöfer, R. and Büchs, J. (2004) A novel parallel shaken bioreactor system for continuous operation. Biotechnology Progress, 20, 1718-1724. doi:10.1021/bp034289a 